\title{
Preservation of Motoneuron Electrotonic Characteristics During Postembryonic Growth
}

\author{
B. Hochner and M. E. Spira \\ Department of Neurobiology, Life Sciences Institute, Hebrew University, Jerusalem, Israel
}

The postembryonic developmental period of the nervous system involves, among other processes, an increase in the dimensions of individual neurons. In the present study we examine whether the characteristic electrotonic parameters that underlie the integrative properties of the neuron $(L$, the electrotonic length; $\rho$, dendritic to somatic conductance ratio; and $\tau_{m}$, the membrane time constant) are established before, during, or after the neurons reach their final adult size. A second aspect that we analyze are the mechanisms by which the neurons adjust their electrotonic parameters during the growth period. The dimensions of the soma and dendrites of the phallic motoneurons (Ph.m.n.) of the cockroach, Periplaneta americana, increase during the last postembryonic developmental stages and metamorphosis to adult by a factor of 1.75 and 1.5 , respectively. This increase is not associated with major changes in the morphological outline of the neuron but is associated with a significant decrease in the input resistance $\left(R_{\mathrm{in}}\right)$ by a factor of 6 (from 120 to $20 \mathrm{M} \Omega$ ). The dendritic to somatic conductance ratio $(\rho)$ is maintained constant. Morphological analysis of cobaltfilled neurons reveals that the neurons can be represented by an equivalent cylinder (Rall, 1969). Calculations of the electrotonic length $(L)$ of the equivalent cylinder from measurements of the time constants $\tau_{m}$ and $\tau_{1}$ (Rall, 1969) revealed that $L$ is constant throughout the period of neuronal growth (1.29 $\pm 0.24 \lambda$ in adult, and $1.13 \pm 0.28 \lambda$ in nymph). The mechanism by which the electrotonic parameters are maintained in spite of growth is continuous adjustment of the diameter and length of the various neuronal segments, rather than by changes in the biophysical properties of the neuron. Several implications in relation to mechanisms of integration and input-output relations during growth and development of neurons are discussed in view of our findings.

The integrative properties of adult neurons are determined by a large number of characteristic parameters (Jack et al., 1975; Bullock, 1976, 1979; Redman, 1976; Rall, 1977; Calvin and Graubard, 1979). These parameters include passive and active membrane properties, the properties and the spatial distribution of receptors and presynaptic terminals, and the architecture of the various neuronal segments, i.e., the soma, dendrites, and axon. Changes in these neuronal properties may alter the input-

\footnotetext{
Received Apr. 7, 1986; revised July 1, 1986; accepted July 7, 1986.

We wish to thank Idan Segev and Robert Werman for comments on an earlier version of the manuscript, and Daniel Zeldes for the camera lucida drawings. This work was supported by a grant from the Israel-U.S.A. Binational Science Foundation to M.E.S.

Copyright (C) 1987 Society for Neuroscience $0270-6474 / 87 / 010261-10 \$ 02.00 / 0$
}

output relations of the neuron and thereby produce changes in behavioral patterns.

The physical dimensions of a neuron's soma, axon, and dendrites increase as the adult neuron differentiates from a neuroblast (Jacobson, 1978; Goodman and Spitzer, 1979). Significant increases in the dimensions may take place even after the major architectural outline of the neuron has been established, i.e., after the major dendritic branching pattern is formed (see Altman and Tyrer, 1974; Scheibel, 1979; Rakic and GoldmanRakic, 1982; Yarom and Spira, 1983; Sadler and Berry, 1984; Blagburn et al., 1985). Alterations in the physical dimensions of the various neuronal segments, especially the dendrites, may alter the electrotonic distance of a particular region and thereby change the input-output relations of the neuron.

The characteristic electrotonic length of adult cat spinal motoneurons is between 1-2 space constants (Lux et al., 1970; Nelson and Lux, 1970; Burke and ten Bruggencate, 1971; Iansek and Redman, 1973; Barrett and Crill, 1974a, b; Rall, 1977). It appears that this electrotonic dimension is independent of the actual physical dimensions of the adult neuron (Kernell, 1966; Burke and ten Bruggencate, 1971), i.e., the same electrotonic length is preserved throughout the size spectrum of the motoneurons. A similar conclusion is indicated from studies of invertebrate neurons. Analysis of the morphological and physiological properties of the giant interneurons in the CNS of the cockroach revealed that the major outline of neurites extending from the interneurons in the metathoracic ganglion is established at early postembryonic developmental stages. While the physical dimensions of the neurites and the axon increase significantly throughout the period of growth and in the adult, the electrotonic length of the segment in the ganglion is not altered (Yarom and Spira, 1983). These results indicate that the electrotonic characteristics of a particular neuronal type are established before the neurons reach their maximal physical dimensions. In other words, it appears that the passive integrative properties of the neuron are not altered during the phase of increase in physical dimensions.

In the present study we examine and correlate changes in the morphological dimensions of motoneurons with their cable properties during the late postembryonic developmental stages. In particular, we examine whether the characteristic electrotonic length $(L)$, the dendritic to somatic conductance ratio $(\rho)$, and the membrane time constant $\left(\tau_{m}\right)$ of a given group of neurons are established prior to their final physical growth, or whether they are established only after the neuron reaches its final size. Distinguishing between these possibilities is of great importance in understanding the manner by which the integrative properties of a neuron are acquired during development. 
The phallic motoneurons (Ph.m.n.) of the CNS of the cockroach (Periplaneta americana) were selected for this study. These neurons are large enough to allow intracellular recording and stimulation throughout various postembryonic developmental stages and can be morphologically characterized by intracellular injections of cobalt ions. Furthermore, the output pattern of the Ph.m.n. pool changes from a random firing pattern at early nymphal stages to a characteristic bursting activity in the adult (Roeder, 1967; Spira and Hochner, 1978).

Our study shows that the general morphological outline of the Ph.m.n. is established during the early stages of postembryonic development. The diameter of the somata, the length of the major dendrites, and the field occupied by the dendrites, however, are markedly increased during the postembryonic developmental period. Despite the significant changes in the morphological dimensions of the neurons, their cable properties are not altered. Our results indicate that the physical growth of the various neuronal segments must be under controls that allow the maintenance of constant electrotonic length throughout postembryonic development.

\section{Materials and Methods}

Animals. Cockroaches (Periplaneta americana) were cultured in our laboratory as previously described (Spira et al., 1976; Spira and Yarom, 1983). At least 7 developmental stages (nymphal stages) have been identified in our culture. The nymphal stages are defined in this paper by the number of molts prior to metamorphosis to the adult form (Spira and Yarom, 1983). For example, adult minus one (Ad-1) defines the last nymphal stage. The present study was performed on adults and nymphal stages $\mathrm{Ad}-4$ to $\mathrm{Ad}-1$.

Preparation. The ventral nerve cord of adult and nymphal cockroaches was isolated as previously described (Spira et al., 1969). The phallic nerves were identified by tracing them from the last abdominal ganglion $\left(\mathrm{A}_{6}\right)$ to the phallic musculature in the adult and to the buds of the phallic apparatus in nymphal stages (Snodgrass, 1935; Roeder et al., 1960; Roeder, 1967; Grossman and Parnas, 1973). The nerves were carefully exposed and then transected close to the muscles in adults and close to the buds of the phallic organs in nymphs. The isolated preparation was pinned ventral side up in a small chamber.

Solutions. Experiments were performed with continuous flow of physiological solution at a rate sufficient to exchange the volume of the experimental chamber once every minute. The physiological solution contained the following: $214 \mathrm{~mm} \mathrm{NaCl}, 3.1 \mathrm{~mm} \mathrm{KCl}, 9 \mathrm{mM} \mathrm{CaCl}_{2}, 10$ mm glucose, $80 \mathrm{~mm}$ sucrose, and $5 \mathrm{~mm}$ Tris $\mathrm{HCl}$. The $\mathrm{pH}$ was adjusted to $7.4-7.6$. Bath temperature was between $20-26^{\circ} \mathrm{C}$.

Electrophysiology. To facilitate penetration of the Ph.m.n. by microelectrodes, the ventral surface of ganglion $A_{6}$ was desheathed. Recording from and stimulation of phallic nerves was done by suction electrodes. For current injection and voltage recordings from Ph.m.n. somata, we used glass microelectrodes filled with $4 \mathrm{M} \mathrm{K}$-acetate. The electrodes used for adult Ph.m.n. had resistances of 20-30 M 2 and, for nymphal Ph.m.n., 30-50 M $\Omega$. The voltage-current relationship and the membrane time constant of the Ph.m.n. were determined in adults by insertion of 2 microelectrodes into a motoneuron soma, one for current injections and the other for voltage recording. In nymphal stages, as well as in some experiments on aduits, only 1 electrode was used for both current injection and voltage recording (WPI electrometer M4-A). For all the experiments we selected electrodes with constant resistance throughout the entire range of current injections. To prevent possible bias of the results due to varying amounts of electrode damage to the motoneurons, we analyzed data from neurons in which the resting potential was at least $-45 \mathrm{mV}$ throughout the experiment.

Analysis of the electrophysiological experiments. The input resistance $\left(R_{\mathrm{in}}\right)$ was calculated from the voltage-current relations in the linear range of membrane resistance, i.e., between $-65 \mathrm{mV}$ to about $-90 \mathrm{mV}$. The membrane time constant, $\tau_{m}$, and the first equalizing time constant, $\tau_{1}$, were calculated using the "peeling" procedure of Rall (1969; Lux et al., 1970; Burke and ten Bruggencate, 1971). Square hyperpolarizing current pulses were injected into the cell body. The rate of voltage development $(d V / d t)$ was plotted against time on a semilogarithmic scale and $\tau_{m}$ and $\tau_{1}$ were obtained from this graph. Using these time constants, the electrotonic length $(L)$ of the neuron was calculated from the equation of Rall $(1969,1977)$ :

$$
L=\pi /\left(\tau_{m} / \tau_{1}-1\right)^{1 / 2} .
$$

This equation is valid if $\rho$, the ratio of dendritic to somatic conductance, is larger than 5 and if the cell can be represented as a cylinder (Rall, 1969, 1977; Jack et al., 1975). As will be shown later, $\rho>18$ and the dendrites appear to be adequately represented as a single cylinder.

Morphology of the motoneurons. The 3-dimensional structure of Ph.m.n. was studied by intracellular injection of cobalt ions into the somata following the methods of Pitman et al. (1972; see also Castel et al., 1976; Yarom and Spira, 1983). Glass microelectrodes filled with 1 $\mathrm{M} \mathrm{CoCl}{ }_{2}$ with resistances ranging between $20-60 \mathrm{M} \Omega$ were used. Cobalt ions were iontophoretically injected by $5-50 \mathrm{nA}$ positive impulses lasting $0.1-1 \mathrm{sec}$ at $0.5-5 \mathrm{~Hz}$. After $10-40 \mathrm{~min}$ of injection, the preparation was washed for 5-30 min and then immersed in physiological solution containing $\left(\mathrm{NH}_{4}\right)_{2} \mathrm{~S}$. The concentration of $\left(\mathrm{NH}_{4}\right)_{2} \mathrm{~S}$ was gradually increased to $0.5 \%$. The preparation was thoroughly washed after 5-15 min of incubation in $\left(\mathrm{NH}_{4}\right)_{2} \mathrm{~S}$ and fixed in Carnoy solution $(60 \%$ ethanol, $10 \%$ acetic acid, $30 \%$ chloroform). Intensification of the cobalt sulfide precipitate was done following the method described by Bacon and Altman (1977). The tissue was dehydrated and cleared using methylbenzoate. Two-dimensional reconstruction of the stained neurons was done by camera lucida retracing.

We have also stained some of the preparations with cobalt using the back-filling method. The nervous system was isolated and placed in physiological solution. The cut ends of the phallic nerves were immersed in a small chamber containing $100 \mathrm{mM} \mathrm{CoCl}_{2}$ in distilled water for 12 $24 \mathrm{hr}$ at $4^{\circ} \mathrm{C}$. The preparation was then processed as described above.

Measurements of somatic and dendritic dimensions were made directly from the preparation. The resolution of our measurements was found to be accurate in the $0.6 \mu \mathrm{m}$ range. Therefore, we have limited quantitative description of dendritic morphology to branches with diameters equal to or larger than $0.6 \mu \mathrm{m}$.

The processing of the preparation from fixation to clearing reduced the dimensions of ganglion $A_{6}$. The reduction in dimensions occurs symmetrically and equally in all directions. The total shrinkage of ganglion $A_{6}$, measured in both adult and nymphal ganglia, was found to be about $30 \%$ in both. Study of the changes in soma dimensions during this procedure revealed a similar reduction in size. Since the main dendritic branches remain undistorted linear structures after the procedure, we assume that the dendrites shrink in a similar proportion. Quantitative morphological data appearing in the present paper were not corrected for these artifacts.

\section{Results}

\section{General morphology of phallic motoneurons}

The morphology of the Ph.m.n. was studied in adult and nymphal stages (Ad-3 and Ad-2) in whole mounts of intracellularly stained motoneurons. Despite a significant increase in the dimensions of the soma and dendrites, the main structural outlines of the motoneurons are not altered during the postembryonic developmental period.

The motoneuron somata are located at the caudo-ventral surface of the 6 th abdominal ganglion (Fig. $1 A$ ). The dendritic tree extends rostrally from the soma into the neuropil of $A_{6}$ and consists of several branches, which can be divided into dorsal (Dd) and ventral (Dv) groups (Fig. 1, $A, D$ ). The dorsal dendrites are connected to the soma by a neurite $(\mathrm{N}$; Fig. $1 D)$ and may consist of 5-9 major branches that ramify extensively along the dorsal portion of the ganglion (Fig. 1, B-E). The ventral dendrites emerge from the same neurite and consist of 1-2 main branches that ramify along the ventral portion of the ganglion. The axonal segment (Ax; Fig. $1 D$ ) of the motoneuron emerges from the region of the junction of the neurite and the dorsal dendrite. Examination of cobalt-filled Ph.m.n. reveals that the basic structure of adult and nymphal neurons is similar. Small variability in the total number of major dendrites can be de- 


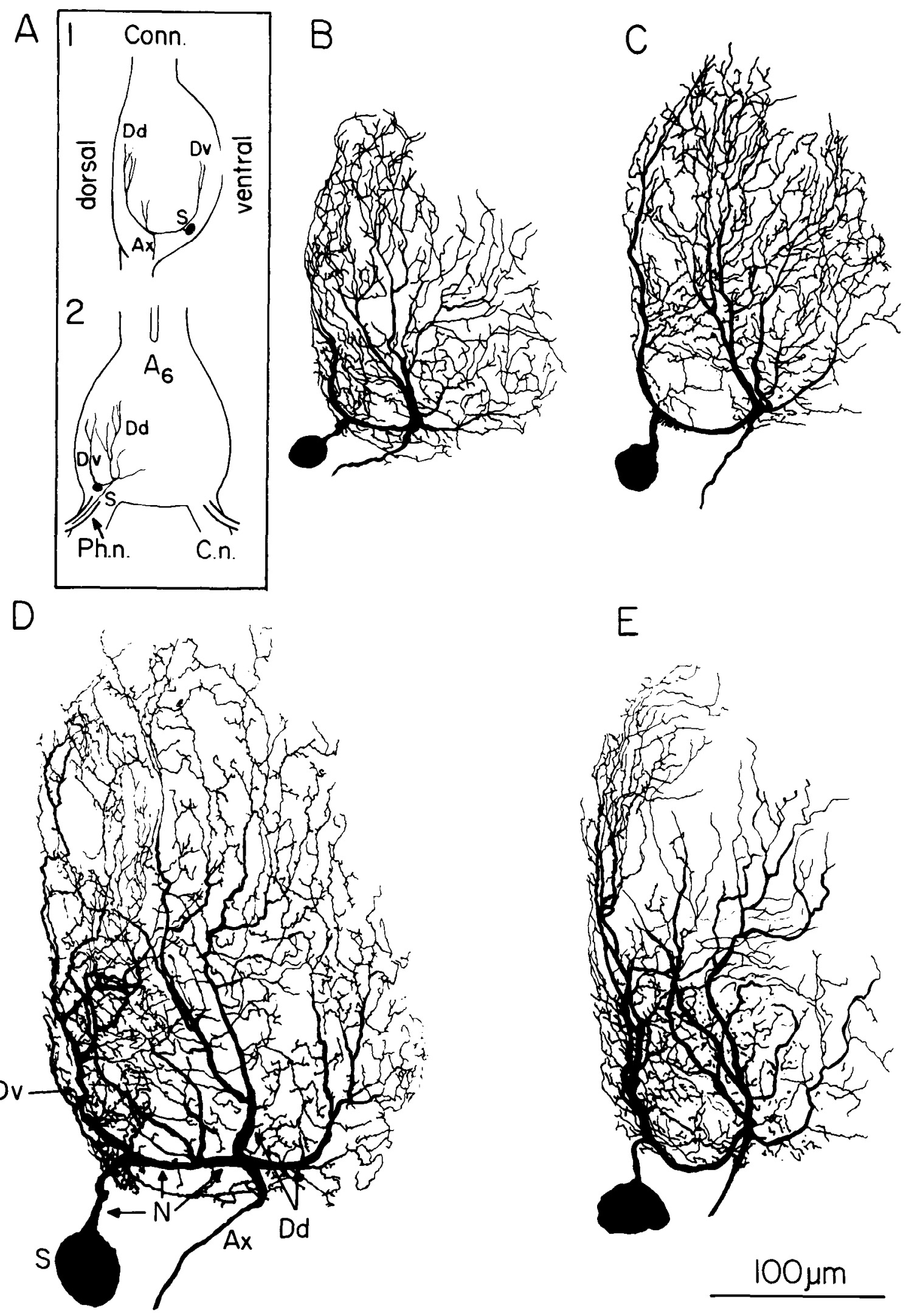

Figure 1. Phallic motoneuron morphology. $A$, Schematic diagram showing the location of a phallic motoneuron soma $(S)$, its dorsal and ventral dendrites $(D d, D v)$, and axon $(A x)$ within the last abdominal ganglion $\left(A_{6}\right)$. Lateral view $\left(A_{1}\right)$; ventral view $\left(A_{2}\right)$. C.n., Cercal nerve, $P h . n$., phallic nerve. $B-F$, Camera lucida tracing of cobalt-filled phallic motoneurons as viewed from the ventral surface of the ganglion. $B$ and $C$, Camera lucida tracing of nymphal phallic motoneurons (stage Ad-2). $D$ and $E$, Adult phallic motoneurons. (Abbreviations in $D$ as in $A ; N$ is the neurite connecting the soma and the most distal dendrites.) Note that the major outline of the neurons is similar in adult and nymphal stages. The dimensions of the soma and dendrites, however, are significantly larger in adults. 


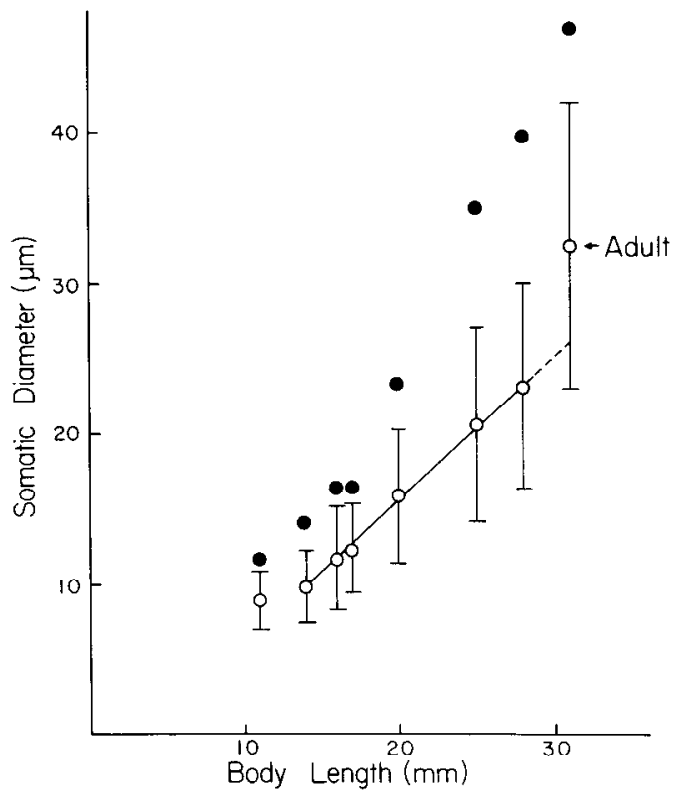

Figure 2. Increase in phallic motoneuron diameter during the postembryonic developmental period. The averaged somatic diameter as revealed by cobalt back-filling is plotted against the body length (empty circles). Each point represents the averaged diameter of at least 15 somata; the SD are represented by the vertical bars. The diameters of the largest cells are indicated by the closed circles. The somatic diameter increased linearly with respect to body length. Note the relatively sharp increase in soma diameter during the metamorphosis to adult stage.

tected in both adult and nymphal Ph.m.n. (Fig. 1, B-E). The average number of major dendrites (with a diameter larger than $0.6 \mu \mathrm{m}$ ) emerging from the neurite $(\mathrm{N})$ is $7.85 \pm 1.68(\mathrm{SD})$ in adults and $6.0 \pm 0.82$ in nymphs. The difference between adults and nymphs is significant $(p<0.05)$, which may be attributed to the resolution of the method used by us to identify major dendrites. Whereas the diameter of the major dendrites of adult $\mathrm{Ph} . \mathrm{m} . \mathrm{n}$. is larger than $0.6 \mu \mathrm{m}$, some of the major dendrites in nymphs are smaller than $0.6 \mu \mathrm{m}$ and therefore may not be defined as such.

The dimensions of the neurons increase gradually during postembryonic developmental stages. The soma diameter increases linearly by a factor of 2.3 (Fig. 2) during nymphal stages Ad 4 to Ad -1 . During the transition between the last nymphal stage and adult (metamorphosis), a sudden increase in the diameter of the Ph.m.n. is observed (Fig. 2). The dimensions of the dendrites increase during development as well (Fig. 1). Measurements of the maximal length of major dendrites from their base to the very tip in stages $\mathrm{Ad}-2, \mathrm{Ad}-3$, and adult reveal that their length increases by a factor of 1.5 (Table 1 ).

The major conclusions from the morphological observations are that the dimensions of the soma and dendrites increase during postembryonic development, while the major morphological outlines of the motoneurons are not altered in a significant way.

\section{Characterization of Ph.m.n. electrical properties}

The experiment of Figure 3 illustrates an example of the voltagecurrent relations of adult (triangles and circles) and nymphal (squares) Ph.m.n. The voltage-current relationships of both the adult and the nymphal neurons are linear in the range of -55 to $-90 \mathrm{mV}$. Further hyperpolarization of the soma reveals the phenomenon of anomalous rectification in the adult (Fig. 3, insert and open symbols in the graph).

Examination of the changes in input resistance of Ph.m.n. (in the linear range of membrane resistance) during the course of postembryonic development reveals that $R_{\text {in }}$ decreases during

Table 1. Morphological parameters of adult and nymph phallic motoneurons

\begin{tabular}{cccccccccc} 
Stage & $\begin{array}{l}D_{S} \\
(\mu \mathrm{m})\end{array}$ & $\begin{array}{c}l_{N} \\
(\mu \mathrm{m})\end{array}$ & $\begin{array}{c}D_{N} \\
(\mu \mathrm{m})\end{array}$ & $\begin{array}{l}l_{D} \\
(\mu \mathrm{m})\end{array}$ & $n$ & $\begin{array}{c}\sum_{1}^{N} D_{i}^{3 / 2} \\
(\mu \mathrm{m})^{3 / 2}\end{array}$ & $\begin{array}{c}D_{E} \\
(\mu \mathrm{m})\end{array}$ & $\begin{array}{c}D_{E v} \\
(\mu \mathrm{m})\end{array}$ & $\begin{array}{c}D_{E d} \\
(\mu \mathrm{m})\end{array}$ \\
\hline Adult & 25.1 & 117 & 2.4 & 280 & 9 & 28.1 & 9.2 & 4.0 & 7.4 \\
& 38.1 & 83 & 4.9 & 250 & 7 & 36.4 & 11.0 & 4.9 & 8.7 \\
& 24.3 & 127 & 4.9 & 300 & 11 & 30.1 & 9.7 & 4.6 & 7.4 \\
& 40.5 & 95 & 4.9 & 315 & 7 & 46.0 & 12.8 & 5.1 & 10.6 \\
& 30.0 & 93 & 3.2 & 302 & 6 & 26.6 & 8.9 & 3.2 & 7.6 \\
& 37.3 & 111 & 4.1 & 315 & 8 & 50.6 & 13.7 & 6.2 & 10.7 \\
Mean & 32.4 & 133 & 3.2 & 277 & 7 & 29.0 & 9.4 & 4.9 & 6.9 \\
\pm SD & \pm 6.4 & 108.4 & 3.94 & 291.3 & 7.85 & 35.26 & 10.67 & 4.70 & 8.47 \\
Nymph & 12.4 & 73 & 1.9 & 187 & 6 & 5.4 & 3.1 & 1.2 & 2.5 \\
& 18.6 & 72 & 1.9 & 189 & 5 & 10.5 & 4.8 & 1.9 & 4.0 \\
& 18.3 & 82 & 1.8 & 201 & 6 & 14.9 & 6.0 & 2.4 & 5.0 \\
& 20.7 & 82 & 1.8 & 201 & 5 & 12.5 & 5.4 & 2.1 & 4.5 \\
& 23.2 & 96 & 2.5 & 220 & 6 & 20.1 & 7.4 & 3.1 & 6.0 \\
& 18.6 & 59 & 1.9 & 157 & 7 & 9.9 & 4.6 & 1.5 & 4.0 \\
& 18.6 & 78 & 3.1 & 208 & 7 & 14.5 & 5.9 & 2.7 & 4.7 \\
Mean & 18.6 & 77.4 & 2.13 & 194.7 & 6.00 & 12.54 & 5.31 & 2.13 & 4.38 \\
\pm SD & \pm 6.4 & \pm 11.3 & \pm 0.49 & \pm 20.0 & \pm 0.82 & \pm 4.63 & \pm 1.34 & \pm 0.66 & \pm 1.08
\end{tabular}

$D_{S}$, cell body diameter; $l_{N}$, neurite length; $D_{N}$, neurite diameter; $l_{D}$, maximal length of the dendrites as measured from the base of the dendrite to its tip; $n$, total number of dorsal and ventral major dendrites that extend from the neurite (the counting of dendrites was limited by the resolution to those with a diameter larger than $0.6 \mu \mathrm{m}$ ); $D_{E}$, equivalent dendritic diameter as given by $\left(\sum_{1}^{N} D_{i}^{3 / 2}\right)^{2 / 3} . D_{i}$ is the diameter of each major dendrite. $D_{E v}$ and $D_{E d}$ are the equivalent dendritic diameters of the major dorsal and ventral dendrites.

The differences between adults and nymphs are significant in all parameters $(t$ test, $p<0.01$ except for $n, p<0.05$ ). 


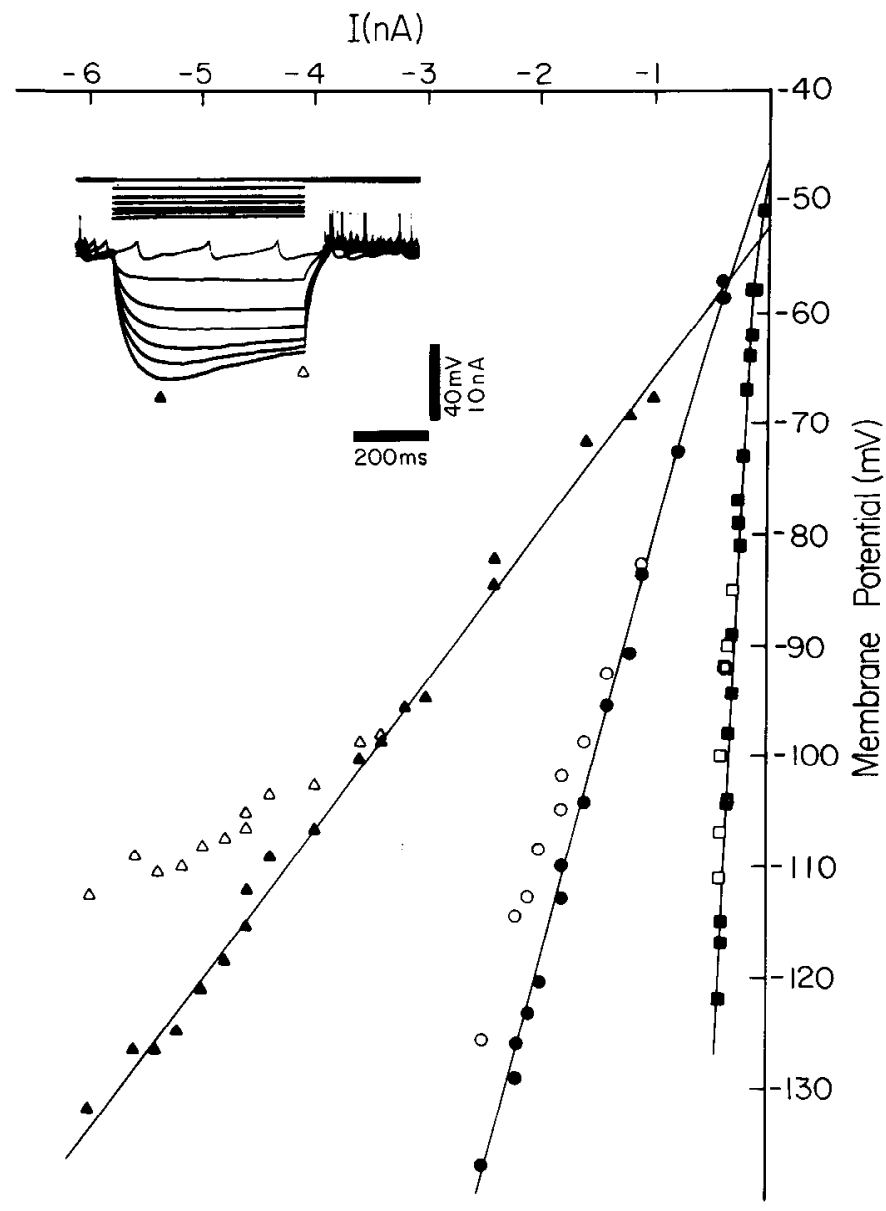

Figure 3. Current-voltage relationships of phallic motoneurons. The insert illustrates the voltage responses to current steps in an adult phallic motoneuron (upper trace, current; lower trace, voltage). Anomalous rectification appears when the membrane is hyperpolarized by about $40 \mathrm{mV}$. The results of similar experiments on adult Ph.m.n. (triangles and circles) and nymphal phallic motoneuron at stage Ad - 3 (squares) are plotted. Filled symbols represent the maximal voltage for a given current pulse; open symbols represent the voltage recorded at the end of the current pulse (steady-state voltage). Note that the Ph.m.n. membrane behaves as a linear resistor in the range of -55 to $-90 \mathrm{mV}$.

development (Fig. 4). The decreased $R_{\text {in }}$ could in principle be attributed to changes in 2 parameters: (1) an increase in the surface area of the neuron, and/or (2) a decrease in the specific membrane resistance $\left(R_{m}\right)$ and/or axoplasmic resistance $\left(R_{i}\right)$. The linear relation between the average $R_{\text {in }}$ and the average reciprocal value of the somatic surface area $(1 / S)$ indicates that the reduction in $R_{\text {in }}$ with growth can be entirely accounted for by the increase in somatic dimensions (Fig. 4). It is interesting to note that the standard deviations of $R_{\text {in }}$ and the SDs of $1 / S$, at each developmental stage, maintain the same linear relation as the average values of $R_{\text {in }}$ and $1 / S$ (dashed lines of Fig. 4 indicate SD). Thus, the variability in $R_{\text {in }}$ within a given developmental stage can also be accounted for by the variability in Ph.m.n. somatic dimensions.

It is possible to explain the linear relation between $R_{\mathrm{in}}$ and $1 / S$ throughout development by assuming that Ph.m.n. soma and the dendrite are in effect electrically isolated from each other by the neurite. If this were true, the measured input resistance would be a function of the somatic surface area and would not

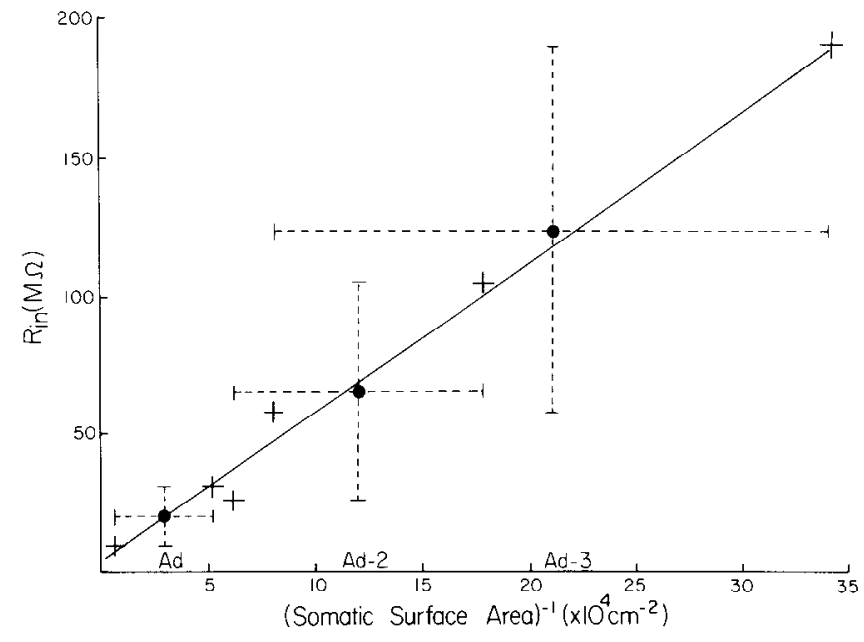

Figure 4. Lincar relationship betwecn phallic motoncuron input resistance $\left(R_{\text {in }}\right)$ and the reciprocal value of somatic surface area $(1 / S)$. The average input resistances of adult phallic motoneurons $(A d)$ and nymphal stages ( $A d-2$ and $A d-3)$ are indicated by the black circles. (Each point was obtained by measurement of $R_{\text {in }}$ of at least 10 neurons and the diameter of at least 15 cells.) The SD of the averaged $R_{\text {in }}$ and $1 / S$ are indicated by the vertical and horizontal broken lines. The pluses $(+)$ correspond to the point of intersection of the SD of $R_{\text {in }}$ and $1 / S$. For further details, see text.

be influenced by the electrical properties of the dendrites. Thus, $R_{\text {in }}=R_{S}$, where $R_{S}$ is the soma resistance. If this were the case, the slope of Figure 4 would be the specific membrane resistance of the soma, giving a value of $560 \Omega \mathrm{cm}^{2}$. Alternatively, if the somatic and dendritic segments of the neurons are not isolated electrically from each other, then the measured input resistance reflects the relative load produced by the 2 compartments. Under these conditions, $R_{\text {in }}=R_{S} /\left(1+R_{S} / R_{D}\right)=R_{S} /(1+\rho)$, where $R_{D}$ is the lumped dendritic resistance and $\rho$ is the ratio of dendritic to somatic conductances. Under these conditions, in order to account for the linear relation between $R_{\text {in }}$ and $1 / S$, it is necessary to assume that $\rho$ is constant throughout development.

We have differentiated between these 2 possibilities by measuring the rate of development of transmembrane voltage to applied square-current pulses into the soma. In an isopotential neuron, the time course of voltage development is described by a single-exponential process, characterized by the membrane time constant $\left(\tau_{m}\right)$. In a nonisopotential neuron the voltage development can be described as an infinite sum of decaying exponents (Rall, 1969).

The rate of development of transmembrane voltage of Ph.m.n. as a function of time is shown on the semilog plot in Figure 5. The log of the derivative of the voltage with respect to time $(d V / d t)$ as a function of time cannot be fitted by a single time constant. At times longer than $20 \mathrm{msec}$, the data can be fitted by a straight line with a slope that represents the reciprocal negative value of the time constant, $\tau_{m}$, of the neuron (38.6 msec; Fig. 5, closed circles). For times shorter than $15 \mathrm{msec}$, the data points deviate from the extrapolated line. Using the "peeling" method of Rall (1969), a second, shorter time constant $(3.3 \mathrm{msec})$ is revealed (Fig. 5, open circles). From these values, $L$ can be calculated using Eq. 1 (Materials and Methods). Table 2 summarizes the results of similar experiments performed on adult and nymphal Ph.m.n. The average time constant, $\tau_{m}$, and the first equalizing time constant, $\tau_{1}$, of adult and nymphal neurons are not statistically different. 


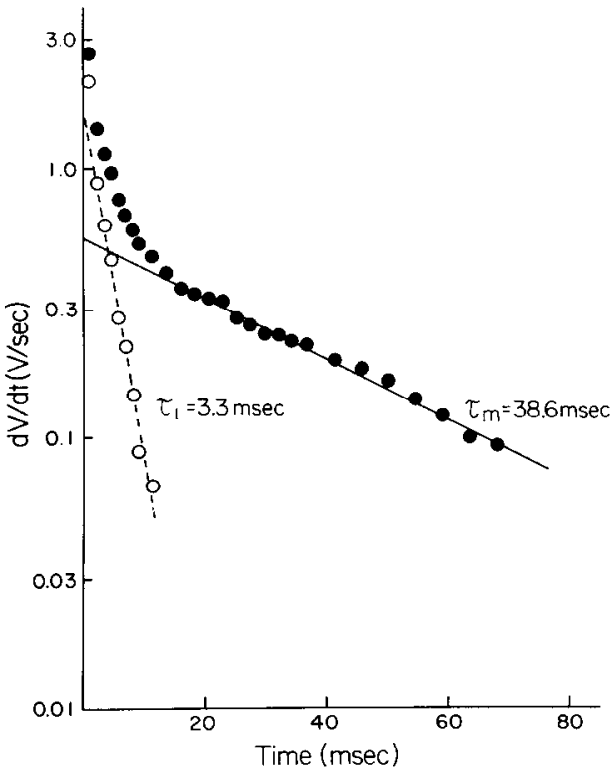

Figure 5. Phallic motoneuron time constants. The membrane time constant $\left(\tau_{m}\right)$ and first equalizing time constant $\left(\tau_{1}\right)$ are calculated from the slopes indicated by the continuous and broken lines, respectively. The values of $d V / d t$ were plotted on log scale. At times longer than 20 msec, the data can be fitted by a straight line (calculated by linear regression). The second, shorter equalizing time constant $\left(\tau_{1}\right)$ is revealed by subtraction of the values derived from the extrapolated line from the data points (open circles).

From these experiments, we conclude that the Ph.m.n. are not isopotential neurons, i.e., the dendrites are electrically long and are not isolated electrically from the soma. The specific mcmbranc resistivity is thus larger than the slope of the line in Figure 4. Finally, the linear relation between $R_{\text {in }}$ and $1 / S$ must indicate a constant relation between the dendritic and somatic conductances (i.e., constant $\rho$ value) throughout the development (see Discussion).

Rall $(1969,1977)$ has shown that the equivalent electrotonic length of an idealized neuron is given by $L=\pi /\left(\tau_{m} / \tau_{1}-1\right)^{1 / 2}$. (In the next section, we will show by quantitative morphological characterization of the Ph.m.n. that these neurons reasonably satisfy the assumptions of Rall's model.) Using the values $\tau_{m}$ and $\tau_{1}$ we have calculated the equivalent electrotonic length $(L)$ of adult and nymphal Ph.m.n. The average electrotonic length of adult neurons is $1.29 \pm 0.24$ (SD) and that of nymphal neurons is $1.13 \pm 0.28$ (Table 2). Thus, it seems that, despite the large increase in the physical dimensions of the neurons, their electrotonic length remains rather constant.

\section{Transformation of the Ph.m.n. to an equivalent cylinder}

The method of calculating the electrotonic length of a neuron, $L$, from $\tau_{m}$ and $\tau_{1}$, was developed for vertebrate motoneurons, in which the dendrites emerge directly from the soma and for neurons with dendritic geometries that obey several other constraints (Rall, 1977). These include (1) the relation $D_{p}^{3 / 2}=$ $\sum_{d}^{N} D_{d}^{3 / 2}$ is satisfied at every point of branching, where $D_{p}$ is the diameter of any parent branch and $D_{d}$ is the diameter of a daughter branch; (2) the electrical distance of all terminal tips from the soma are the same; and (3) all terminal tips end with the same boundary conditions. The cockroach Ph.m.n., as well as other invertebrate neurons, differs significantly from the clas-

\begin{tabular}{llll}
\hline $\begin{array}{l}\text { Table 2. Electrical parameters of adult and nymph phallic } \\
\text { motoneurons }\end{array}$ & \multicolumn{3}{l}{} \\
Stage & $\tau_{m}$ & $\tau_{1}$ & \\
(msec) & $(\mathrm{msec})$ & $L$ \\
\hline Adult & 33.6 & 5.8 & 1.43 \\
& 71.0 & 12.7 & 1.47 \\
& 43.8 & 3.3 & 0.90 \\
& 22.9 & 2.9 & 1.20 \\
& 31.9 & 6.8 & 1.63 \\
& 17.3 & 1.8 & 1.08 \\
Mean \pm SD & 23.5 & 2.9 & 1.18 \\
Nymph & 15.8 & 2.9 & 1.49 \\
& $32.5 \pm 18.1$ & $4.9 \pm 3.5$ & $1.29 \pm 0.24$ \\
& 38.6 & 3.0 & 0.91 \\
& 45.5 & 5.6 & 1.18 \\
& 40.6 & 6.2 & 1.33 \\
& 23.7 & 2.6 & 1.10 \\
& 17.3 & 1.7 & 1.05 \\
& 27.3 & 1.4 & 0.73 \\
& 55.0 & 5.7 & 1.07 \\
Mean \pm SD & 28.8 & 6.4 & 1.68 \\
& $34.6 \pm 12.5$ & $4.1 \pm 2.1$ & $1.13 \pm 0.28$
\end{tabular}

$\tau_{m}$, membrane time constant; $\tau_{1}$, first equalizing time constant; $L$, the electrotonic length of the phallic motoneuron (in space constant units) as calculated from $\tau_{m}$ and $r_{1}$

sical vertebrate neurons in their structural organization (Bullock and Horridge, 1965; Cohen, 1976). The major difference is that in invertebrates the soma is spatially separated from the dendrites and axon by a segment called a neurite. The structural organization of the dendritic trees is also different from that in classical vertebrate motoneurons.

We undertook an analysis of the morphology of the Ph.m.n. in order to evaluate whether the assumptions, on which the use of the Rall equation (Eq. 1) is based, are satisfied by the Ph.m.n.

The first question we addressed is whether the neurite connecting the soma and the dendrite electrically isolates these 2 compartments. It is conceivable that the resistance and capacity introduced by the thin neurite may attenuate the current spread from the soma into the dendrite. For such a case, the derivation of the equalizing time constants on which Eq. 1 is based is incorrect. In order to tackle this problem, we first estimated the electric length of the neurite and then calculated the coupling time constant.

The average length of neurites in adult Ph.m.n. is $108 \mu \mathrm{m}$ (range, 83-133 $\mu \mathrm{m}$; Table 1) and in the nymphal stages Ad 2 and $\mathrm{Ad}-3,77 \mu \mathrm{m}$ (range, 59-96 $\mu \mathrm{m}$ ). The average diameter is $3.94 \mu \mathrm{m}$ (range, $2.4-4.9 \mu \mathrm{m}$ ) and $2.13 \mu \mathrm{m}$ (range, 1.8-3.1) in adults and nymphs, respectively. We have calculated the neurite electrotonic length by assuming that the specific membrane resistance $\left(R_{m}\right)$ is the same in adults and nymphs and that it ranges between $20-50 \mathrm{~K} \Omega \mathrm{cm}^{2}$. The value was calculated from $\tau_{m}$, assuming that the membrane capacity is $1 \mu \mathrm{F} / \mathrm{cm}^{2}$ (see Table 2). The axial resistivity is assumed to be $130 \Omega \mathrm{cm}$ (Yarom and Spira, 1983). The calculated electrotonic length of the neurite in nymphs and adults is similar, ranging between 0.055 and $0.080 \lambda$ for the above ranges of $R_{m}$ values.

From that estimate we may conclude that essentially most of the current injected at the soma reaches the dendrites. Hence, the neurite can be regarded as a coupling resistor, $R_{N}$, between the soma and the dendrites. 


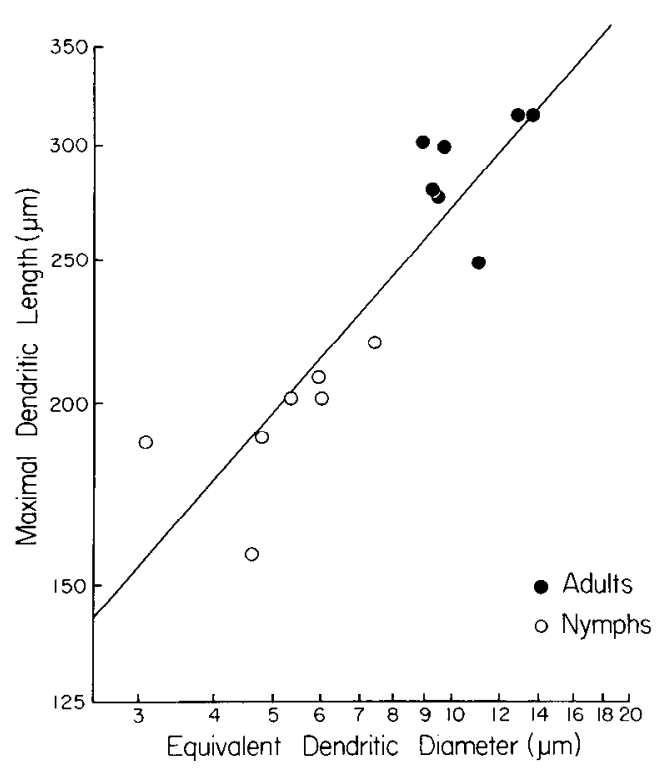

Figure 6. Correlation between the logarithms of measured length of the Ph.m.n. dendrites and the logarithm of equivalent dendritic diameter. (Data from Table 1.) The equivalent diameter is given by $\left(\sum_{1}^{N} D_{i}^{3 / 2}\right)^{2 / 3}$ and represents the diameter of the equivalent dendritic cylinder (see Rall, 1977). The slope of this relation as calculated by linear regression is 0.47 with correlation coefficient of 0.892 . See text for further details.

Assuming that the neurite can be represented as a coupling resistance, then the parameter that determines to what degree the soma and the dendrites are isolated from one another is $\tau_{N}=R_{N} C_{S}$, where $\tau_{N}$ is the coupling time constant, and $C_{S}$ is the soma capacitance (Rall, 1981). When $\tau_{N}$ is very small relative to $\tau_{m}$, the dendrites can be viewed as emerging directly from the soma. That would indicate that the neurite has no decoupling effect, and the potential at the base of the dendrites follows that of the soma with no delay or attenuation. This is the case for the neurite in both nymphs and adults. The average $\tau_{N}$ in both was calculated to be about $0.3 \mathrm{msec}$. This value is small enough relative to $\tau_{m}$ and $\tau_{1}$ to assume that, despite the presence of a neurite, we can treat the cell as if the dendrites were directly coupled to the soma. It should be noted that these calculations were done for the worst case, where all the dendrites emerge from the distal end of the neurite. This conclusion agrees with the electrophysiological analysis which indicates that the dendrites are not isolated electrically from the cell body.

The second question that we address is to what extent the relation $D_{p}^{3 / 2}=\sum_{1}^{N} D_{d}^{3 / 2}$ is satisfied by the Ph.m.n. The diameters of primary, secondary, and some tertiary dendritic branches of adult neurons and primary and secondary branches of nymphal neurons were measured from whole mounts of cobalt-filled neurons. The ratio $D_{p}^{3 / 2}$ to $\sum_{1}^{N} D_{d}^{3 / 2}$ is very close to unity, $1.12 \pm 0.31(n=27)$ for adult neurons and $1.12 \pm 0.20$ $(n=9)$ for nymphal neurons (see Table 1). Thus, this aspect of dendritic geometry satisfies the necessary rcquircment at lcast for the major branching points.

The morphological reconstruction of the dendrites from the soma to the very tip by measurements of the diameters and length of all daughter branches of a given tree cannot be done accurately. As explained in Materials and Methods, such measurements are limited to a resolution of $0.6 \mu \mathrm{m}$. Therefore, it is impossible to evaluate quantitatively whether the electrical

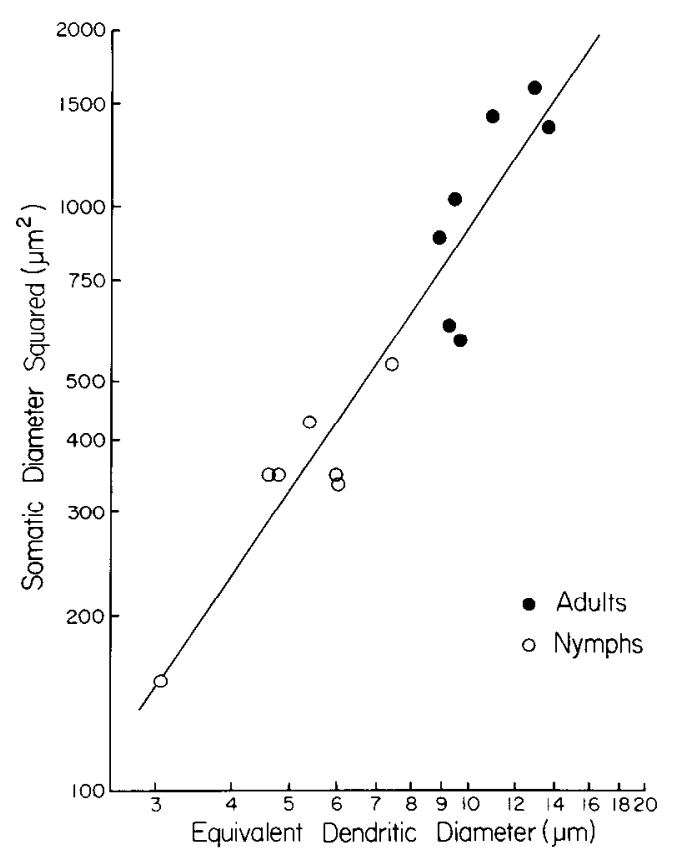

Figure 7. Correlation between logarithm of somatic diameter squared and logarithm of the equivalent dendritic diameter. The slope of this relation is 1.513 with a correlation coefficient of 0.948 . See text for further details.

lengths of all dendritic trees (from soma to terminals, and assuming homogeneous membrane properties) are the same. Nevertheless, there is a clear qualitative correlation between the diameter of the dendrites and their length. Those with small diameters are always the shortest. This impression is strengthened by the result described by Figure 6 (see below). Finally, there is no obvious and direct way to define the boundary properties of the dendritic terminals. There are no physiological or morphological reasons, however, to assume that the dendritic terminals of the Ph.m.n. should not be treated as sealed-end terminals.

These considerations of Ph.m.n. morphology indicate that both adult and nymphal Ph.m.n. may be represented by a single equivalent cylinder with sealed ends, as required by the assumption on which Eq. 1 was developed (Rall, 1969).

\section{Mechanisms underlying the preservation of Ph.m.n. electrical length}

The constancy of the electrotonic length $(L)$ of the neurons, despite the significant elongation of the dendrites in development, requires that either the membrane properties or morphological dimensions of the dendrite are continuously and properly adjusted throughout the development. If we assume that $R_{m} / R_{i}$ is constant throughout development, then the relation between the physical length of the equivalent cylinder $\left(l_{E}\right)$ and its diameter $\left(D_{E}\right)$ is given by

$$
l_{E}=L \lambda=L /\left(D_{E} R_{m} / 4 R_{i}\right)^{1 / 2}
$$

where $\lambda$ is the space constant of the equivalent cylinder. If the constancy in the electrotonic length of adult and nymphal Ph.m.n. can be accounted for by adjustment of diameter and length of each segment in the dendritic tree, and if the number of branches is kept constant during this period of development, then there will be a linear relation between $l_{E}$ and the physical length of 
the dendritic tree $\left(l_{D}\right)\left(l_{E}=K l_{D}\right)$. In this case, the slope of $\log \left(l_{D}\right)$ against $\log \left(D_{E}\right)$ should be 0.5 . Figure 6 shows that this slope is actually 0.47 , with a correlation coefficient of 0.89 . This result strongly suggests that the electrotonic length of the dendrites is maintained during development by continuous morphological matching between the length and diameter of dendrites, and there is no need to assume alteration in specific properties of either the membrane or the axoplasm.

Another relationship that was found to be constant throughout the postembryonic stage is the one between $R_{\text {in }}$ and $1 / S$ (see Fig. 4). If the soma can be regarded as a sphere, and the dendrites as a single cylinder with a sealed end, then their corresponding input resistance is

$$
R_{\mathrm{in}(S)}=R_{m} / \pi D_{S}^{2}
$$

and

$$
R_{\text {in }(D)}=(1 / \pi)\left(4 R_{m} R_{i} / D_{E}^{3}\right)^{1 / 2} \operatorname{coth}(L) .
$$

Assuming that $L, R_{m}$, and $R_{i}$ are constant, then

$$
\rho=R_{\mathrm{in}(S)} / R_{\mathrm{in}(D)}=\mathrm{K}\left(D_{S}{ }^{2} / D_{E}^{3 / 2}\right)
$$

where $\mathrm{K}$ is a constant.

Hence, if the assumption concerning the constancy of $L, R_{m}$, and $R_{i}$ is true, we expect the slope of a plot of $\log \left(D_{S}{ }^{2}\right)$ against $\log \left(D_{E}\right)$ to be 1.5 . Figure 7 shows that this relation for Ph.m.n. is 1.514 , with a correlation coefficient of 0.948 . Thus, these results, which are based exclusively on morphological parameters (i.e., $D_{S}$ and $D_{E}$ ), agree with the conclusions based on electrophysiologic findings, which show that $\rho$ is also constant throughout this postembryonic developmental period of these neurons. Assuming an average $L=1.2, R_{m}=30,000 \Omega \mathrm{cm}^{2}$, and $R_{i}=130 \Omega \mathrm{cm}$, we found that $\rho$ equals 21 in nymphs and 22.3 in adults. These values were calculated using the average parameters for adults and nymphs in Table 1 and the above equations for $R_{\mathrm{in}(S)}$ and $R_{\mathrm{in}(D) \text { * }}$

\section{Discussion}

The major conclusion of the present investigation is that the characteristic electrotonic properties, i.e., the electrotonic length of the neuron $(L)$ and the dendritic to somatic conductance ratio $(\rho)$, are not altered during the postembryonic developmental stages, a period characterized by significant physical growth of the neurons, as well as by changes in the output pattern of the motoneuron pool. Our analysis indicates that the mechanisms responsible for maintenance of constant electrotonic properties of the neurons involve a continuous adjustment of the morphological dimensions of the neuron, rather than by adjustment of the specific electrical properties (i.e., $R_{m}, R_{i}, C_{m}$ ).

\section{Representation of the phallic motoneurons by an equivalent circuit}

The major structural outline of the Ph.m.n. is unchanged during the last 4 postembryonic developmental stages and throughout the metamorphosis to adult. The variability in the number of major branches may be attributed to the fact that we have not identified individual Ph.m.n. but rather identified a pool of neurons. It is reasonable to assume that homologous Ph.m.n. in different individuals reveal less structural variability. It is known from studies of other invertebrates that some structural variability can be detected between identified neurons even in genetically identical individuals (Altman and Tyrer, 1974; Goodman, 1978). In any case, since the variability in major branches is similar within a given nymphal stage and between the various stages, it does not reflect a developmental process. Despite the small variability, the Ph.m.n. share a basic morphological outline. A typical Ph.m.n. can be represented by a morphophysiological equivalent composed of 4 compartments: (1) the soma; (2) the neurite connecting the soma with the dendrites; (3) the ventral dendrite that emerges from the neurite close to the cell body; and (4) the group of dorsal dendrites.

The separation between the cell body and dendrite by the neurite, a typical case for arthropod neurons, may, in extreme cases, isolate the soma from the dendrite electrically. In such cases, analysis of the electrical length by Rall's method cannot be applied. However, as shown in the Results section, in the case of the Ph.m.n. the average electrotonic length of the neurite is less than $0.1 \lambda$ (for adults and nymphs) and the coupling time constant is negligible $(0.3 \mathrm{msec})$. Thus, the neurons can be treated as if the dendrites emerge directly from the cell body.

Measurements of the diameters of the primary, secondary, and tertiary dendritic branches revealed that the $d^{3 / 2}$ ratio between parent and daughter branches is close to unity. Therefore, it is possible to represent the major dendrites as equivalent cylinders (Rall, 1962, 1977). These were lumped together to form a single equivalent cylinder. Such transformation requires that all the major dendrites should have the same electrical length. Since there is a qualitative correlation between the diameter and length of the major dendrites, this transformation seems to be justified. Thus, we conclude that the Ph.m.n. can be represented as an equivalent cylinder with an average electrotonic length $(L)$ of about $1.2 \lambda$ (range, $0.73-1.68 \lambda$ ). This $L$ is not statistically different in nymphs and adults.

To evaluate the validity of this equivalent model, in the following paragraph we recalculate the expected input resistance from the physical dimensions of the 4 compartments equivalent and the specific membrane resistances $R_{m}$ as obtained from the measurements of $\tau_{m}$. For these approximations, we assume membrane capacity of $1 \mu \mathrm{F} \mathrm{cm}{ }^{2}$ (Jack, 1979). The somatic input resistance $\left(R_{S}\right)$ is given by

$$
R_{S}=R_{m} / \pi D_{S}^{2} .
$$

The resistances of the equivalent cylinders representing the ventral and dorsal dendrites were calculated from the finite sealedend cable equation (Rall, 1977) and are given by

$$
R_{D}=(1 / \pi)\left(4 R_{m} R_{i} / D_{E}^{3}\right)^{1 / 2} \operatorname{coth}(L) .
$$

The coupling resistance introduced by the neurite was calculated by

$$
R_{N}=4 R_{i} l_{N} / \pi D_{N}^{2} .
$$

The total input resistance $R_{\text {in }}$ as measured from the soma is given by

$$
\frac{1}{R_{\mathrm{in}}}=\frac{1}{R_{S}}+\frac{1}{R_{\mathrm{Dv}}}+\frac{1}{R_{\mathrm{Dd}}+R_{N}} .
$$

Taking $R_{m}$ to be $30,000 \Omega \mathrm{cm}^{2}$ and $R_{i}$ to be $130 \Omega \mathrm{cm}$ and using the average dimensions in Table 1 , the calculated input resistance, $R_{\text {in }}$, of the adult Ph.m.n. is $46.4 \mathrm{M} \Omega$ and for nymphs 127.0 M . These values are twice as large as the experimental results. However, if the dimensions of the soma, dendrites, and neurite are corrected for the shrinkage artifact (see Materials and Methods), then the calculation gives the following values for the input resistances: $28 \mathrm{M} \Omega$ for the adult and $80 \mathrm{M} \Omega$ for the nymph. These values are much closer to those of the ex- 
perimental results (Fig. 4). The relation between dendritic and somatic conductances $(\rho)$ was calculated from the 4-compartment equivalent model; it was found to be about 18 in both nymphs and adult neurons. (The ratio is only slightly affected by the shrinkage artifact.)

The correspondence between the physiological experiments and the calculations based on the morphological dimensions of the neurons support the conclusions that $L, \rho$, and specific electrical properties are indeed preserved throughout the developmental period studied. As we do not have to assume that $R_{m}$, $R_{w}$, and $C_{m}$ are changed, we conclude that the mechanism by which the neuron maintains its constant functional dimensions must be by a continuous adjustment of the various morphological parameters (length and diameter of each compartment). The high correlation (Fig. 7) between the somatic surface area and $D_{E}^{3 / 2}$ (where $D_{E}$ is the diameter of the equivalent cylinder), which accounts for the constant $\rho$, as well as the constant relation (Fig. 6) between $D_{E}$ and $l_{D}$ (where $l_{D}$ is dendritic length), which accounts for the constant electrical length $(L)$, further supports this conclusion.

\section{Physiological significance of the short $\mathrm{L}$}

Despite the elaborate branching pattern of their dendrites, the Ph.m.n. are quite compact in terms of their electrical length. It is interesting to note in this respect that they are similar to various mammalian neurons (Lux and Pollen, 1966; Lux et al., 1970; Nelson and Lux, 1970; Burke and ten Bruggencate, 1971; Iansek and Redman, 1973; Barrett and Crill, 1974a; Turner and Schwartzkroin, 1980; Brown et al., 1981; Johnston, 1981; Durand et al., 1983; Stafstrom et al., 1984, 1985). The Ph.m.n., as do vertebrate neurons, receives a large number of converging synaptic inputs. A short electrical length may imply that the neurons cannot discriminate among the various inputs. Electrotonic length of 1.2 implies that a steady-state voltage generated at the soma will attenuate to $55 \%$ of its amplitude when it reaches the tip of the dendrites. Likewise, a synchronized input to all the dendritic tips will decrement to $55 \%$ when it reaches the soma (Rall, 1962). It should be noted, however, that a single synaptic input to the tip of only 1 dendrite will be attenuated by a much larger factor (Rall and Rinzel, 1973; Rinzel and Rall, 1974). For example, a steady-state synaptic input to the tips of only 1 out of 7 major dendrites in the average Ph.m.n. would be attenuated to about $10 \%$ of its value when reaching the cell body. Thus, despite the short $L$ value, and because of the extensive dendrite branching, the attenuation of a single input may be very large, and allow the neuron to "discriminate" between various inputs as a function of their location in the dendrites.

\section{Physiological significance of preservation of $\mathrm{L}$ and reduction in input resistance during development}

The input resistance $\left(R_{\text {in }}\right)$ of the Ph.m.n. is reduced as the physical dimensions of the neurons are increased. This decrease implies that synaptic current $\left(I_{\text {sy }}\right)$ generated by a given synapse will produce a smaller voltage drop if the synaptic terminal maintains its relative position on the dendrites. To maintain a constant postsynaptic voltage, $I_{\text {sy }}$ must be adjusted to the changes in $R_{\text {in }}$. An alternative is that the relative position of the synapse on the dendrite with respect to the cell body will change. In both cases the maintenance of synaptic input efficacy (as measured by the voltage generated by a given synapse at the cell body or spike initiation zone) requires a coordination between the changes in the pre- and postsynaptic elements. In this regard, it is interesting to mention that some of the identified synaptic inputs to the Ph.m.n. maintain a rather constant amplitude and shape throughout the developmental period (Ad - 3 to Ad) (B. Hochner and M. E. Spira, unpublished observations). Thus, in this case, the synaptic inputs maintain constant efficacy despite the decrease in $R_{\text {in }}$. The mechanisms by which the presynaptic terminal and postsynaptic elements interact during cell growth are not known.

We wish to point out that in cases where $L$ and $\rho$ are kept constant by morphological adjustments of each segment, there is a linear relation between the surface area and input conductance $\left(G_{\text {in }}=1 / R_{\text {in }}\right)$ of each segment. Therefore, matching between the increase in $G_{\text {in }}$ during development and $I_{\text {sy }}$ to give the same voltage may be achieved simply if the synaptic area grows together with the nonsynaptic membrane in such a way that the fraction of area occupied by the synapse remains constant. In this way, the increase in $I_{\text {sy }}$ will exactly compensate for the decrease in $R_{\text {in }}$ to yield the same synaptic voltage. This reasoning will be true if the density of receptors, ionic channels, and releasing sites is maintained. It is interesting to note that if the density and spatial distribution of the various voltage dependent channels is maintained during growth, the input-output relation of a given neuron will be preserved in spite of growth.

The characteristic electrical parameters of a neuron, as defined by Rall (1977), serve as a very useful tool for comparison of some integrative parameters of neurons. However, since the membrane resistance of most neurons does not behave as a fixed resistor, but rather reveals voltage-dependent changes, $L$ must be viewed as a dynamic parameter. Namely, the physiological ranges of $L$ for a given neuron may be shorter or longer depending on the transmembrane voltage induced by the physiological activity (Yarom and Spira, 1983; Segev and Parnas, 1985). Although $L$ of the Ph.m.n. may be much larger when the neurons are depolarized (due to decreased membrane resistance), the conclusion that $L$ is preserved throughout development is still valid. Our experiments (not described in detail in this paper) show that the Ph.m.n. membrane resistance of nymphs and adults reveal identical dependency on transmembrane voltage.

Our study shows that preservation of the Ph.m.n. electrotonic parameter is achieved by morphological adjustments of the length and diameters of the various neuronal compartments. The cellular mechanisms by which such precise control is executed are unknown.

\section{References}

Altman, J. S., and N. M. Tyrer (1974) Insect flight as a system for the study of the development of neuronal connections. In Experimental Analysis of Insect Behaviour, L. Barton Browne, ed., pp. 159-179, Springer-Verlag, New York.

Bacon, J. P., and J. S. Altman (1977) A silver intensification method for cobalt-filled neurons in wholemount preparations. Brain Res. 138: 359-363.

Barrett, J. N., and W. E. Crill (1974a) Specific membrane properties of cat motoneurons. J. Physiol. (Lond.) 239: 301-324.

Barrett, J. N., and W. E. Crill (1974b) Influence of dendritic location and membrane properties on the effectiveness of synapses on cat motoneurons. J. Physiol. (Lond.) 239: 325-345.

Blagburn, J. M., D. J. Readle, and D. B. Sattelle (1985) Development of chemosensitivity of an identified insect interneurone. J. Neurosci. 5: 1167-1175.

Brown, T. H., R. A. Fricke, and D. H. Perkel (1981) Passive electrical constants in three classes of hippocampal neurons. J. Neurophysiol. 46: 812-827. 
Bullock, T. H. (1976) In search of principles in neural integration. In Simpler Networks and Behavior, J. C. Fentress, ed., pp. 52-60, Sinauer, Sunderland, MA.

Bullock, T. H. (1979) Evolving concepts of local integrative operations in neurons. In The Neurosciences: Fourth Study Program, F. O. Schmitt and F. G. Worden, eds., pp. 43-49, MIT Press, Cambridge, MA.

Bullock, T. H., and G. A. Horridge (1965) Structure and Function in the Nervous Systems of Invertebrates, Freeman, San Francisco.

Burke, R. E., and G. ten Bruggencate (1971) Electronic characteristics of alpha motoneurones of varying size. J. Physiol. (Lond.) 212: 120.

Calvin, W. H., and K. Graubard (1979) Styles of neuronal computation. In The Neurosciences: Fourth Study Program, F. O. Schmitt and F. G. Worden, eds., pp. 513-524, MIT Press, Cambridge, MA.

Castel, M., M. E. Spira, I. Parnas, and Y. Yarom (1976) Ultrastructure of a region of low safety factor in an unhomogeneous giant axon of the cockroach. J. Neurophysiol. 39: 900-908.

Cohen, M. J. (1976) Cellular events in the evolution of behaviour. In Simpler Networks and Behaviour, J. C. Fentress, ed., pp. 39-51, Sinauer, Sunderland, MA.

Durand, D., P. L. Carlen, N. Gurevich, A. Ho, and H. Kunov (1983) Electrotonic parameters of rat dentate granule cells measured using short current pulses and HRP staining. J. Neurophysiol. 50: 10801096.

Goodman, C. S. (1978) Isogenic locusts: Genetic variability in the morphology of identified neurons. J. Comp. Neurol. 182: 681-705.

Goodman, C. S., and N. C. Spitzer (1979) Embryonic development of identified neurons: Differentiation from neuroblast to neuron. Nature 280: 208-214.

Grossman, Y., and I. Parnas (1973) Control mechanisms involved in the regulation of the phallic neuromuscular system of the cockroach Periplaneta americana. J. Comp. Physiol. 82: 1-21.

Iansek, R., and S. J. Redman (1973) An analysis of the cable properties of spinal motoneurones using brief intracellular current pulse. J. Physiol. (Lond.) 234: 613-636.

Jack, J. (1979) An introduction to linear cable theory. In The Neurosciences: Fourth Study Program, F. O. Schmitt and F. G. Worden, eds., pp. 423-437, MIT Press, Cambridge, MA.

Jack, J. J. B., D. Noble, and R. W. Tsien (1975) Electrical Current Flow in Excitable Cells, Oxford U. P., London.

Jacobson, M. (1978) Developmental Neurobiology, Plenum, New York.

Johnston, D. (1981) Passive cable properties of hippocampal CA3 pyramidal neurons. Cell. Mol. Neurobiol. 1: 41-55.

Kernell, D. (1966) Input resistance, electrical excitability, and size of ventral horn cells in cat spinal cord. Science 152: 1637-1640.

Lux, H. D., and D. A. Pollen (1966) Electrical constants of neurons in the motor cortex of the cat. J. Neurophysiol. 29: 207-220.

Lux, H. D., P. Schubert, and G. W. Kreutzberg (1970) Direct matching of morphological and electrophysiological data in cat spinal motoneurons. In Excitatory Synaptic Mechanisms, P. Andersen and J. K. S. Jansen, eds., pp. 189-198, Universitetsforlaget, Oslo.

Nelson, P. G., and H. D. Lux (1970) Some electrical measurements of motoneuron parameters. Biophys. J. 10: 55-73.

Pitman, R. M., C. D. Tweedle, and M. J. Cohen (1972) Branching of central neurons: Intracellular cobalt injection for light and electron microscopy. Science 176: 412-414.

Rakic, P., and P. S. Goldman-Rakic, eds. (1982) Development and modifiability of the cerebral cortcx. Ncurosci. Res. Prog. Bull. 20: 429-611.

Rall, W. (1962) Theory of physiological properties of dendrites. Ann. NY Acad. Sci. 96: 1071-1092.
Rall, W. (1969) Time constants and electrotonic length of membrane cylinders and neurons. Biophys. J. 9: 1483-1508.

Rall, W. (1977) Core conductor theory and cable properties of neurons. In Handbook of Physiology, Sect. 1, The Nervous System, Vol. 1, Cellular Biology of Neurons, E. R. Kandel, ed., pp. 39-97, American Physiological Society, Bethesda, MD.

Rall, W. (1981) Functional aspects of neuronal geometry. In Neurones Without Impulses: Their Significance for Vertebrate and Invertebrate Nervous Systems, A. Roberts and B. M. H. Bush, eds., pp. 223-254, Cambridge U. P., Cambridge, UK.

Rall, W., and J. Rinzel (1973) Branch input resistance and steady attenuation for input to one branch of a dendritic neuron model. Biophys. J. 13: 648-688.

Redman, S. J. (1976) A quantitative approach to integrative function of dendrites. In International Review of Physiology: Neurophysiology II, Vul. 10, R. Porter, ed., pp. 1-36, University Park Press, Baltimore, MD.

Rinzel, J., and W. Rall (1974) Transient response in a dendritic neuron model for current injected at one branch. Biophys. J. 14: 759-790.

Roeder, K. D. (1967) Nerve Cells and Insect Behaviours, Harvard U. P., Cambridge, MA.

Roeder, K. D., L. Tozian, and F. A. Weiant (1960) Endogenous nerve activity and behaviours in the mantis and the cockroach. J. Insect Physiol. 4: 45-62.

Sadler, M., and M. Berry (1984) Remodelling during development of the Purkinje cell dendritic tree in the mouse. Proc. R. Soc. London [Biol.] 211: 349-368.

Scheibel, A. B. (1979) Development of axonal and dendritic neuropil as a function of evolving behaviours. In The Neurosciences: Fourth Study Program, F. O. Schmitl and F. G. Worden, eds., pp. 381-398, MIT Press, Cambridge, MA.

Segev, I., and I. Parnas (1985) Nonlinear cable properties of the giant axon of the cockroach Periplaneta americana. J. Gen. Physiol. 85: $729-741$.

Snodgrass, R. E. (1935) Principles of Insect Morphology, McGrawHill, New York.

Spira, M. E., and B. Hochner (1978) Analysis of the neuronal circuit that controls the phallic nerve activity of the cockroach Periplaneta americana during development. Isr. J. Med. Sci. 14: 1095.

Spira, M. E., and Y. Yarom (1983) Functional elimination of afferent pathways and decreased safety factor during post embryonic development of cockroach giant interneurons. Dev. Brain Res. 8: 311 360.

Spira, M. E., I. Parnas, and F. Bergman (1969) Organization of the giant axons of the cockroach Periplaneta americana. J. Exp. Biol. 50: 615-627.

Spira, M. E., Y. Yarom, and I. Parnas (1976) Modulation of spike frequency by regions of special axonal geometry and by synaptic inputs. J. Neurophysiol. 39: 882-899.

Stafstrom, C. E., P. C. Schwindt, and W. E. Crill (1984) Cable properties of layer $\mathrm{V}$ neurons from cat sensorimotor cortex in vitro. J. Neurophysiol. 52: 278-289.

Stafstrom, C. E., P. C. Schwindt, and W. E. Crill (1985) Letter to the editor. J. Neurophysiol. 54: 1084.

Turner, D. A., and P. A. Schwartzkroin (1980) Steady-state electrotonic analysis of intracellularly-stained hippocampal neurons. J. Neurophysiol. 44: 184-199.

Yarom, Y., and M. E. Spira (1983) Morphological and electrophysiological properties of the giant interneurons during the post embryonic development of the cockroach CNS. Dev. Brain Res. 8: 321334. 